\title{
Exceptional manifestation of polyautoimmunity in a very young girl - a case report
}

\author{
AGNIESZKA MROCZKOWSKA-JUCHKIEWICZI', JACEK POSTĘPSKI ${ }^{2}$ EDYTA OLESIŃSKA², \\ PAULINA KRAWIEC ${ }^{l}$ ELŻBIETA PAC-KOŻUCHOWSKA ${ }^{l}$
}

${ }^{1}$ Department of Paediatrics, Medical University of Lublin, Lublin, Poland

${ }^{2}$ Department of Paediatric Pulmonology and Rheumatology, Medical University of Lublin, Lublin, Poland

\begin{abstract}
Polyautoimmunity is defined as the presence of more than one autoimmune disease in a single patient. The exact pathogenic mechanisms responsible for the coexistence of distinct autoimmune diseases within an individual have not been clearly explained. We report a case of a very young girl with the extremely rare co-existence of four distinct autoimmune diseases i.e. juvenile idiopathic arthritis, type 1 diabetes mellitus, coeliac disease and autoimmune hepatitis, recognized based on validated international classification criteria. The best to our knowledge there has been no case reporting coexistence of these particular four disorders in an individual. Moreover, all these diseases occurred during first three years of life, which also cause that case unique. Molecular studies of human leukocyte antigen (HLA) class II in our patient showed the presence of the HLA DRBI*01, HLA DRBI*03, HLA DQB1*02, HLA $D Q B 1 * 05$ molecules, which may suggest immunogenetic links between those autoimmune diseases. The presented case highlights the importance of active screening for other autoimmune diseases, if a patient with one autoimmune disease manifests with new or nonspecific symptoms.
\end{abstract}

Key words: juvenile idiopathic arthritis, autoimmune hepatitis, celiac disease, type 1 diabetes, polyautoimmunity.

(Cent Eur J Immunol 2017; 42 (1): 107-110)

\section{Introduction}

Polyautoimmunity is defined as the presence of more than one overt autoimmune disease in a single patient, while overlapping syndromes means the partial presence of symptoms of diverse autoimmune diseases. Coexistence of three or more autoimmune disorders is called multiple autoimmune syndrome [1].

The exact pathogenic mechanisms responsible for the coexistence of distinct autoimmune diseases within an individual have not been clearly explained. However, genetic susceptibility, environmental factors, deregulation of the immune response and hormonal impairment may be implicated in the polyautoimmunity. Diverse outcomes of genes underlying similar immunogenic mechanisms indicate the possible shift from one disease to another [1]. Enhanced knowledge of the pathogenesis of autoimmune diseases and advances in the molecular studies allowed to define the genetic factors of susceptibility to autoimmune diseases including both Human Leukocyte Antigen (HLA) and non HLA loci (e.g. CTLA4, PTPN22, TNFA, IL-6, STAT4) [2, 3].

We report a case of a girl with four distinct autoimmune diseases i.e. juvenile idiopathic arthritis, type 1 diabetes mellitus, celiac disease and autoimmune hepatitis, which manifested in the first three years of life. It should be highlighted, that the coexistence of four autoimmune disorders is extremely rare particularly in a very young age. Moreover, all these diseases occurred in a very short period of time, which also cause that case unique. The best to our knowledge to date there has been no case reporting coexistence of these particular four disorders in a single patient. However, there have been reports of variable associations between any two of these autoimmune diseases.

\section{Case presentation}

A 20-months-old girl of unrelated, healthy parents was admitted to the Department of Paediatric Pulmonology and Rheumatology with joints pain and swelling lasting for four months. Patient's complaints started with swelling and tenderness of left toe, followed by left ankle, right knee, and right elbow. The past medical history concerning preceding infections, traumas, immunizations and family history of autoimmune diseases was unremarkable.

Laboratory results revealed elevation of inflammatory markers (erythrocyte sedimentation rate $93 \mathrm{~mm} / \mathrm{h}$; normal $<10 \mathrm{~mm} / \mathrm{h}$, C-reactive protein $107 \mathrm{mg} / \mathrm{l}$; normal < $5 \mathrm{mg} / \mathrm{l}$ ) and positive anti-nuclear antibodies with a titre of $1: 1280$.

Correspondence: Agnieszka Mroczkowska-Juchkiewicz, Department of Paediatrics, Medical University of Lublin, Chodzki 2,

20-093 Lublin, Poland, e-mail: aga-juchkiewicz@ poczta.onet.pl

Submitted: 30.03.2016; Accepted: 13.05.2016 
There were no presence of anti-extractable nuclear antigen antibodies, human leukocyte antigen B27 and rheumatoid factor. Moreover, infection and malignancy were excluded. The girl was diagnosed with oligoarticular juvenile idiopathic arthritis. The treatment with naproxen, methotrexate with the bridge therapy additionally prednisone used as bridge therapy, was started. Moreover, the girl underwent right knee puncture with intra-articular long-lasting glucocorticosteroid injection.

Two months after the initial diagnosis, at the age of 22 months, our patient presented polyuria, polydipsia, weight loss and vulvitis. Laboratory results showed hyperglycaemia with the highest value of $409 \mathrm{mg} / \mathrm{dl}$ (normal serum glucose level $<100 \mathrm{mg} / \mathrm{dl}$ ), metabolic acidosis and glycosuria and ketonuria. Moreover, elevated haemoglobin A1c (14.45\%; normal 4.8-5.9\%) and fructosamine levels (734 $\mu \mathrm{mol} / \mathrm{l}$; normal $205-285 \mu \mathrm{mol} / \mathrm{l})$ were stated. Type 1 diabetes mellitus with ketoacidosis was diagnosed. The therapy with insulin and diabetic diet was implemented. The patient continued to receive methotrexate and prednisone.

After three consecutive months (at the age of 2 years and 1 month) our patient was admitted to the hospital because of persistent vomiting once daily independently on meals for several weeks. Physical examination revealed no abnormalities. Laboratory tests showed normal complete blood count and blood serum chemistry with parameters of well-controlled diabetes. However, positive IgA anti-endomysial antibodies (IgA EmA) with a titre of $1: 1280$ were stated. Based on that result, our patient was qualified to the gastroscopy, which revealed flattened Kerckring's folds with scalloping. In the histological examination infiltration with intraepithelial lymphocytes in the submucosa and total duodenal villous atrophy was stated, thus confirming the diagnosis of celiac disease classified as Marsh stage 3c. The strict gluten-free diet was administered.

At the age of 2 years and 6 months hypertransaminasemia was stated (ALT $807 \mathrm{U} / 1$; normal $<50 \mathrm{U} / 1$ and AST - $542 \mathrm{U} / \mathrm{l}$; normal < $60 \mathrm{U} / \mathrm{l})$ with elevation of $\alpha 2$ - and $\beta$-globulins in the serum protein electrophoresis. Laboratory tests showed normal levels of bilirubin, total proteins, albumins, immunoglobulins (IgG, IgA, IgM) and coagulation tests. Based on the serological tests viral hepatitis caused by hepatitis B virus, hepatitis $\mathrm{C}$ virus, cytomegalovirus, Ebstein-Barr virus, enteroviruses and herpes simplex virus were excluded. The serum level of creatine kinase, $\alpha_{1}$-antitrypsin, ceruloplasmin, copper and 24-hour urinary copper and sweat test were within the normal range. Thus, muscular dystrophy, $\alpha_{1}$-antitrypsin deficiency, cystic fibrosis and Wilson's disease we excluded. Further tests revealed positive anti-nuclear antibodies with a titre of $1: 160$ and positive anti-smooth muscle antibodies with a titre of $1: 80$, and the absence of liver kidney microsomal type 1 antibodies. Ultrasound examination of the liver was normal. Due to the fact that adverse effect of methotrexate could not be excluded, the administration of methotrexate was ceased and the treatment was continued solely with prednisone. In the face of unclear reason of hypertransaminasemia, the percutaneus liver biopsy was performed. The histological examination revealed chronic hepatitis G3S1 according to the Batts and Lewis scale. Based on International Autoimmune Hepatitis Group Criteria, autoimmune hepatitis was recognized and treatment with azathioprine and prednisone was started.

Simultaneously with the hypertransaminasemia, laboratory results revealed hyperthyroidism. However, autoimmune causes of that disorder were excluded. Additionally, treatment with thiamazole and propranolole was implemented.

In our patient, Human Leukocyte Antigen (HLA) class II typing by the Polymerase Chain Reaction-Micro-Sequence Specific Primer (PCR-Micro-SSP) showed the presence of the HLA DRB $1 * 01$, HLA DRB $1 * 03$, HLA DQB1*02, HLA DQB1*05 molecules.

During the three-year follow-up our patient was in a good general condition. We observe a low activity of juvenile idiopathic arthritis, well-controlled type 1 diabetes mellitus, euthyreosis and normalization of liver function tests. She is treated with insulin, thiamazole, azathioprine and low doses of glucocorticosteroids and complied with an appropriate diabetic and gluten-free diet.

\section{Discussion}

Autoimmune disorders result from the mounting immune response against self antigens. It leads to the damage of tissues expressing specific self-antigens and the generation of auto-antibodies. However, the aetiology of autoimmune disorders is complex and multifactoral.

Distinct autoimmune diseases often present similar clinical picture and course. The coexistence of several autoimmune disorders in a single patient as well as occurrence of distinct autoimmune diseases within the members of one family was noted. Moreover, autoimmune disorders affect predominantly women $[4,5]$.

In recent years, considerable progress has been made in the understanding of the autoimmune disorders' pathogenesis, due to the development of molecular biology and genetics. Genome wide association studies identified susceptibility genes to many autoimmune diseases [6].

In our patient, the first manifestation of polyautoimmunity was juvenile idiopathic arthritis. This is the most common chronic arthritis in childhood, diversified according to the pathogenesis and the clinical course. A phenomenon of the co-occurrence of juvenile idiopathic arthritis with other autoimmune diseases is well-established. The association of juvenile idiopathic arthritis with both HLA class I and II genes has been showed [2]. The single-nucleotide polymorphism of the PTPN 22 gene (encoding protein tyrosine phosphate receptor type 22) was found in the associations of juvenile idiopathic arthritis with type 1 diabetes mel- 
litus, systemic lupus erythematosus and Graves-Basedov disease $[2,6]$.

Albers et al. showed that $4 \mathrm{q} 27$ region encoding interleukin 2 and 2, previously reported to be associated with celiac disease, rheumatoid arthritis, type 1 diabetes mellitus and the psoriatic arthritis, may be also associated with juvenile idiopathic arthritis [6].

The co-occurrence of juvenile idiopathic arthritis and type 1 diabetes mellitus is known and has been previously described [4, 6-9]. In our patient, symptoms of type 1 diabetes mellitus occurred after the recognition of juvenile idiopathic arthritis.

Numerous genetic studies have demonstrated more than fifty susceptibility genes, which indicate the possibility of co-occurrence of autoimmune diseases including connective tissue diseases and the digestive system diseases with celiac disease [9].

Another autoimmune disorder in our patient was celiac disease. Celiac disease is a chronic immune-mediated inflammation of the small intestine elicited by the gluten ingestion in genetically susceptible individuals. The co-existence of celiac disease and type 1 diabetes mellitus is well-established. The prevalence of celiac disease in patients with type 1 diabetes ranges from 0.6 to $16.4 \%$ depending on different studies [10]. Children with type 1 diabetes recognized before 6 years of age had greater risk of celiac disease, compared with children diagnosed after that age [11]. The association between celiac disease and type 1 diabetes results from an overlap of susceptibility genes to both diseases. The HLA-DR3/DQ 2 antigen is expressed in 90-95\% patients with celiac disease and $55 \%$ of those with type 1 diabetes mellitus. About 5-10\% of remaining celiac patients present HLA-DQ 8 antigen, which is also attributed to the increased risk of type 1 diabetes [10]. Taking into consideration these facts, screening testing for celiac disease should be offered to individuals with type 1 diabetes to avoid potential complications of delayed diagnosis. However, it should be highlighted that HLA associated genes predisposing to celiac disease may be found in $30-40 \%$ healthy people. The final diagnosis is based on the positive celiac-specific antibody levels and characteristic histological changes (villous atrophy and crypt hyperplasia) in the duodenal biopsy [12]. However, HLA typing could be useful in the celiac recognition. Moreover, novel potential criteria for celiac disease have been suggested i.e. four out of following five criteria (typical symptoms, high titer of celiac specific antibodies, positive HLADQ2/DQ8, enteropathy on small bowel biopsy and response to gluten free diet) should be fulfilled to recognize celiac disease [12]. Recent studies have shown the association of variation in NLRP3 and interleukin 2 gene polymorphisms in pathomechanism of autoimmune diseases including celiac disease [11]. Genetic testing in our patient revealed the HLA DRB1*01, DRB $1 * 03$, DQB $1 * 02$, $\mathrm{DQB} 1 * 05$ haplotype, which is a genetic risk factor for coexistence of the celiac disease and type 1 diabetes mellitus.
In our patient, eight months after the initial diagnosis with juvenile idiopathic arthritis, hypertransaminasemia was found in laboratory results. Elevation of liver function tests may result from drugs hepatotoxicity, thrombotic incidents or coexisting liver autoimmune disorders [13]. In our patient based on detailed diagnostic process including liver biopsy, autoimmune hepatitis was confirmed. Although liver diseases are not characteristic in juvenile idiopathic arthritis, both disorders may coexist. Moreover, hepatomegaly is one of the diagnostic criteria of systemic juvenile idiopathic arthritis [13].

Several liver disorders have been reported in celiac patients. The most common liver disorder in celiac disease, occurring in $40 \%$ of patients, is non-specific hepatitis manifested with isolated mild hypertransaminasemia. Usually, introduction of gluten-free diet leads to normalization of liver function tests. However, celiac disease may be also associated with autoimmune liver diseases, including autoimmune hepatitis, primary sclerotic cholangitis or primary biliary cirrhosis [14]. In that case, gluten-free diet is insufficient in the treatment of liver disease. Thus, testing for autoimmune liver diseases should be considered in a patient with celiac disease and hypertransaminasemia. In our patient juvenile idiopathic arthritis and celiac disease increased the risk of autoimmune hepatitis.

\section{Conclusions}

We presented a case of extremely rare co-existence of juvenile idiopathic arthritis, type 1 diabetes mellitus, celiac disease and autoimmune hepatitis in the very young girl. This is an exceptional manifestation of polyautoimmunity suggesting common genetic susceptibility and immune mechanisms. The present case underlines the importance of active screening for other autoimmune diseases, if a patient with one autoimmune disease manifests with new or nonspecific symptoms. It should be also noted that early recognition of polyautoimmunity is crucial for clinicians in the patient's management and formulating prognosis.

\section{Consent}

Written informed consent was obtained from the patient's parents for publication of this case report.

The authors declare no conflict of interest.

\section{References}

1. Anaya JM (2014): The diagnosis and clinical significance of polyautoimmunity. Autoimmun Rev 13: 423-426.

2. Prahalad S, Glass DN (2008): A comprehensive review of the genetics of juvenile idiopathic arthritis. Pediatr Rheumatol Online J 8: 6-11.

3. Thompson SD, Sudman M, Ramos PS, et al. (2010): The susceptibility loci juvenile idiopathic arthritis shares with 
other autoimmune diseases extend to PTPN2, COG6, and ANGPT1. Arthritis Rheum 62: 3265-3276.

4. Neuhausen SL, Steele L, Ryan S (2008): Co-occurrence of celiac disease and other autoimmune diseases in celiacs and their first-degree relatives. J Autoimmun 31: 160-165.

5. Criswell LA, Pfeiffer KA, Lum RF, et al. (2005): Analysis of families in the multiple autoimmune disease genetics consortium (MADGC) collection: the PTPN22 620W allele associates with multiple autoimmune phenotypes. Am J Hum Genet 76: 561-571.

6. Albers HM, Kurreeman FA, Stoeken-Rijsbergen G (2009): Association of the autoimmunity locus $4 \mathrm{q} 27$ with juvenile idiopathic arthritis. Arthritis Rheum 60: 901-944.

7. Drachal E, Lisowicz L, Surdej B, Korczowski B (2009): Coexistence of autoimmune diseases and diabetes mellitus type 1 in children in south-eastern Poland. Przegl Ped 39: 248-251.

8. Hinks A, Martin P, Flynn E, et al. (2010 ): Investigation of type 1 diabetes and coeliac disease susceptibility loci for association with juvenile idiopathic arthritis. Ann Rheum Dis 69: 2169-2172.

9. Pehlić M, Vrkić D, Skrabić V, et al. (2012): IL12RB2 gene is associated with the age of type 1 diabetes onset in Croatian family Trios. PLoS One 7: e49133.

10. Scaramuzza A, Mantegazza C, Bosetti A, Zuccotti GV (2013): Type 1 diabetes and celiac disease: The effects of gluten free diet on metabolic control. World J Diabetes 4: 130-134.

11. Samasca G, Sur G, Lupan I, et al. (2014): Celiac disease as an autoimmune condition. Cent Eur J Immunol 39: 396-399.

12. Rachisan AL, Pirvan A, Miu N, et al. (2016): Is HLA Typing a Diagnostic Tool in Celiac Disease? Int J Celiac Dis 4: 9-10.

13. Nobili V, Devito R, Comparcola D, et al. (2005): Juvenile idiopathic arthritis associated with autoimmune hepatitis type 2 . Ann Rheum Dis 64: 157-158.

14. Sima H, Hekmatdoost A, Ghaziani T, et al. (2010): The prevalence of celiac autoantibodies in hepatitis patients. Iran J Allergy Asthma Immunol 9: 157-162. 